\title{
The cases treated with lung cancer in the hospital center "xhaferr kongoli" in the period time of 2013-2015
}

Introduction: Lung cancer is one of the most common and serious types of cancer. Cancer that begins in the lungs is called primary lung cancer. Cancer that spreads to the lungs from another place in the body is known as secondary lung cancer. There are two main types of primary lung cancer. These are classified by the type of cells in which the cancer starts.

They are:

1. Non-small-cell lung cancer - the most common type, accounting for more than $80 \%$ of cases; can be squamous cell carcinoma, adenocarcinoma or large-cell carcinoma.

2. Small-cell lung cancer - a less common type that usually spreads faster than non-small-cell lung cancer.

Imaging techniques used for the detection, characterisation, staging and follow-up of lung cancer are:

- Conventional chest radiography,

- Computed tomography (CT),

- Magnetic resonance (MR),

- $\quad$ Positron emission tomography (PET).

Methods: This research is descriptive with retrospective components and the data are taken from the book of protocol of the people with Lung Cancer treated in the hospital Center "Xhaferr Kongoli" in the period time of 2013-2015. This study involved 47 patients.

Results: From 47 patients 45 are males (96\%) and 2 are females (4\%). From these 26 patients or $55 \%$ are from the city and 21 patients or $45 \%$ are from village.

The most affected age is 55-64 years old with 26 patients (55\%), over 65 years old with 14 patients (30\%), $45-54$ years old with 7 patients (15\%).

From 47 patients 30 are smokers (64\%) and 17 patients are non-smokers (36\%).

Conclusion: Based on the date obtained from patients treated for lung cancer we conclude that:

1. The most frequently affected age group is 55-64 years old.

2. The most affected sex is male.

3. It is more spread in urban areas than rural ones.

Lung cancer mainly affects smokers versus non-smokers.

KEYWORDS: lung cancer - $\mathrm{x}$-ray $~=$ cells

\section{Introduction}

Lung cancer starts when abnormal cells grow out of control in the lung. They can invade nearby tissues and form tumors. Lung cancer can start anywhere in the lungs and affect any part of the respiratory system. The cancer cells can spread or metastasize, to the lymph nodes and other parts of the body.

Lung cancers are divided into small cell lung cancers (SCLC 20-25\% of cases) and non-small cell lung cancers (NSCLC $70-80 \%$ of cases) based on clinical behaviour and histological appearance. Small cell lung cancers usually grow more quickly and are more likely to spread than non-small cell lung cancer $[1,2]$.

There are three different types of SCLC:

- Small-cell carcinoma (oat cell carcinoma)

- Mixed small-cell/large-cell carcinoma

- Combined small-cell carcinoma

NSCLC is further divided histologically into three main disease subtypes of:

- Squamous cell carcinoma comprises approximately $30 \%$ of lung cancers. These tumours generally arise centrally 
within the lungs inside a large bronchus although they may sometimes be peripheral.

- Adenocarcinoma, representing perhaps $30 \%$ of invasive lesions, tends to occur in more peripheral locations arising from the smaller airways but they can be found centrally in a main bronchus.

- Large cell carcinoma, $10 \%$ of lung cancers, is undifferentiated tumours which lack the diagnostic features of the other subtypes. This is therefore to some extent a default classification, made when other specific histology has been excluded [3-6].

\section{- Histologic classification of NSCLC:}

\section{Squamous cell carcinoma}
a. Papillary
b. Clear cell
c. Small cell
d. Basaloid

\section{Adenocarcinoma}

a. Acinar

b. Papillary

c. Bronchioloalveolar carcinoma

i. Non-mucinous

ii. Mucinous

iii. Mixed mucinous and non-mucinous or indeterminate cell type

d. Solid adenocarcinoma with mucin

e. Adenocarcinoma with mixed subtypes

f. Variants

i. Well-differentiated

fetal adenocarcinoma

ii. Mucinous (colloid) adenocarcinoma

iii. Mucinous cystadenocarcinoma

iv. Signet ring adenocarcinoma

v. Clear cell adenocarcinoma

\section{Large cell carcinoma}

a. Variants

i. $\quad$ Large cell neuroendocrine carcinoma (LCNEC) ii. Combined LCNEC

iii. Basaloid carcinoma

iv. Lymphoepithelioma-like carcinoma

v. Clear cell carcinoma

vi. Large cell carcinoma with rhabdoid phenotype

\section{Adenosquamous carcinoma}

5. Carcinomas with pleomorphic, sarcomatoid or sarcomatous elements

a. Carcinomas with spindle and/or giant cells

b. Spindle cell carcinoma

c. Giant cell carcinoma

d. Carcinosarcoma

e. Pulmonary blastoma

\section{Carcinoid tumor}
a. Typical carcinoid
b. Atypical carcinoid

\section{Carcinomas of salivary gland type}

a. Mucoepidermoid carcinoma

b. Adenoid cystic carcinoma

c. Others.

\section{Unclassified carcinoma}

The etiology, or the root cause of lung cancer, is not always evident. Although tobacco smoking is the primary etiological factor, scientists agree that many lung cancers have no single cause, but are instead the result of a combination of several causes that may include exposure to radon, asbestos, environmental tobacco smoke and exposures to other hazardous materials.

Smoking cigarettes is the single biggest risk factor for lung cancer. It's responsible for more than $85 \%$ of all cases. Tobacco smoke contains more than 60 different toxic substances, which can lead to the development of cancer. These substances are known to be carcinogenic (cancerproducing). While smoking cigarettes is the biggest risk factor, using other types of tobacco products can also increase the risk of developing lung cancer and other types of cancer, such as esophageal cancer and mouth cancer.

Passive smoking or the inhalation of tobacco smoke by non-smokers, who share living or 
working quarters with smokers, also is an established risk factor for the development of lung cancer.

Air pollution from vehicles, industry, and power plants can raise the likelihood of developing lung cancer in exposed individuals [7-9]. Up to $1 \%-2 \%$ of lung cancer deaths are attributable to breathing polluted air and experts believe that prolonged exposure to highly polluted air can carry a risk for the development of lung cancer similar to that of passive smoking.

Patients with lung cancer present with progressive shortness of breath, cough, chest pain/oppression, hoarseness or loss of voice, hemoptysis (mostly with squamous cell carcinoma). Pneumonia is the presenting feature in many patients. Relative to other forms of nonsmall cell lung cancer, adenocarcinoma is more often asymptomatic, being more frequently identified in screening studies or as an incidental radiologic finding. Patients with small cell lung cancer (SCLC) differ in many ways from those with non-small cell lung cancer (NSCLC), in that they often present with symptoms referable to distant metastases. About $10 \%$ of patients with SCLC present with superior vena cava syndrome. Stridor and hemoptysis are rare symptoms in patients with SCLC. Symptoms related to disseminated disease include weight loss, abdominal pain due to involvement of the liver, adrenals and pancreas, and pain due to bone (marrow) metastases [10,11].

Lung cancer is usually first found on a chest $\mathrm{X}$-ray or a CT scan. A chest X-ray of someone with lung cancer may show a visible mass or nodule. This mass will look like a white spot on your lungs, while the lung itself will appear black. In some situations, no further imaging will be necessary when bulky contralateral mediastinal adenopathy is present or when an obvious bony lesion is identified [12]. However, CT scanning of the chest is often needed because of the lack of sensitivity of the chest radiographs in detecting mediastinal lymph node metastases and chest wall and mediastinal invasion. CT can identify specific features in lung nodules that are diagnostic, e.g. arteriovenous fistulae, rounded atelectasis, fungus balls, mucoid impaction and infarcts. High-resolution scanning further refines this diagnostic process. Spiral or helical CT increases the detection rate of nodules $<5 \mathrm{~mm}$ in diameter. Spiral CT with a bolus injection of intravenous iodinated contrast medium affords "dynamic scanning".

Magnetic resonance imaging (MRI) is becoming more available but pressure on MRI scanning time is so intense that it is usually used for problem solving and where administration of contrast media is contraindicated. The advantages MRI has over CT include: better soft tissue contrast, multiplanar imaging capability, and therefore useful for superior sulcus tumours and evaluation of the aortopulmonary window. MRI is also useful in the assessment of mediastinal and chest wall invasion by virtue of its ability to determine fat-stripe invasion and involvement of the diaphragm and spinal canal. MRI is poorly tolerated by claustrophobic patients and is contra-indicated in patients with indwelling electromagnetic devices and some prosthetic heart valves.

T1-weighted sequences are used for the visualization of fat planes and improved spatial resolution. T2-weighted sequences are useful for detection of high-signal tumour infiltration. Gadolinium enhancement can further enhance the diagnostic yield [13].

Positron emission tomography (PET) scanning is a new imaging modality whose role in the assessment of lung cancer is still being determined. Its advantage over other modalities lies in its sensitivity in detecting malignancy and its ability to image the entire body in one examination. PET scanning detects malignancy in focal pulmonary opacities with a sensitivity of $96 \%$, specificity of $88 \%$ and an accuracy of $94 \%$ in lesions of $\geq 10 \mathrm{~mm}$. However, compared to CT, PET has poorer spatial resolution, which precludes it from accurate anatomical assessment of primary tumour status. False-positive PET findings in the lung are seen in tuberculous infection, histoplasmosis and rheumatoid lung disease. False negatives are seen with carcinoid tumours, bronchoalveolar carcinoma and lesions $<10 \mathrm{~mm}$ in size. PET is more accurate than CT in the detection or exclusion of mediastinal nodal metastases. The main disadvantage for PET is the lack of availability and relatively high cost of each examination. However, decision analysis models indicate that combined use of CT and PET imaging for evaluating focal pulmonary lesions is the most cost-effective and useful strategy in determining patient management with a pretest likelihood of having 
a malignant nodule of $0.12-0.69$. PET is more accurate than conventional studies in detecting recurrent lung cancer and appears to be superior in distinguishing persistent or recurrent tumour from fibrotic scars [14].

Fiberoptic bronchoscopy allows macroscopic examination of the respiratory tree up to most of the subsegmental bronchi and biopsies associated to bronchial aspiration and brushing. Biopsies of bone, liver, lymph node (mediastinoscopy), skin and adrenal gland may also be used for diagnosis if they are metastatically involved. Pulmonary function tests are performed if surgery seems possible. Serum tumour markers are not routinely recommended. Because of its central location squamous cell carcinoma is readily diagnosed by bronchoscopic biopsy and/ or brush and/or sputum cytology. Fluorescence bronchoscopy may be useful for assessing the extent of associated intraepithelial neoplasia. For peripheral lesions transthoracic CT guided fine needle aspiration biopsy is now generally preferred. Due to common central location, small cell carcinoma is often diagnosed via bronchoscopically retrieved histologic and cytologic samples and to a lesser extent sputum cytology. Small peripheral lesions are often subjected to fine needle aspiration biopsy, transbronchial biopsy, or sometimes wedge resection for initial diagnosis.

Small cell lung cancer stages are classified in two ways:

1. Limited stage: The cancer is found in one lung, sometimes including nearby lymph nodes.

2. Extensive stage: Cancer has spread to the other lung, the fluid around the lung (the pleura) or to other organs in the body.

Non-small cell lung cancer staging uses the TNM system:

- Tumor (T) describes the size of the original tumor.

- Lymph node $(\mathrm{N})$ indicates whether the cancer is present in the lymph nodes.

- Metastasis (M) refers to whether cancer has spread to other parts of the body, usually the liver, bones or brain.

- Stages of non-small cell lung cancer:

- Occult stage non-small cell lung cancer:
Cancer cells are found in sputum, but no tumor can be found in the lung by imaging tests or bronchoscopy or the tumor is too small to be checked.

- Stage $O$ non-small cell lung cancer: Cancer at this stage is also known as carcinoma in situ. The cancer is tiny in size and has not spread into deeper lung tissues or outside the lungs.

- Stage I non-small cell lung cancer: Cancer may be present in the underlying lung tissues, but the lymph nodes remain unaffected.

- Stage II non-small cell lung cancer: The cancer may have spread to nearby lymph nodes or into the chest wall.

- Stage III non-small cell lung cancer: The cancer is continuing to spread from the lungs to the lymph nodes or to nearby structures and organs, such as the heart, trachea and esophagus.

- Stage IV non-small cell lung cancer: The cancer has metastasized throughout the body and may now affect the liver, bones or brain.

\section{Materials and methods}

This research is descriptive with retrospective components and the data are taken from the book of protocol of the people with Lung Cancer treated in the hospital Center "Xhaferr Kongoli" in the period time of 2013-2015 (the first 5 months of 2015) [15]. This study involved 47 patients (TABLE 1 ).

\section{Results}

As can be seen in FIGURE 1, the most affected age is 55-64 years old with 26 patients (55\%), over 65 years old with 14 patients $(30 \%)$, 45-54 years old with 7 patients (15\%). In 2013, 1 patient is 45-54 years old, 12 patients are 5564 years old and 5 patients are over 65 years old. In 2014, 3 patients are 45-54 years old, 13 patients are 55-64 years old and 6 patients are

\begin{tabular}{|c|c|}
\hline Years & $\begin{array}{c}\text { The number of patients } \\
\text { with lung cancer }\end{array}$ \\
\hline 2013 & 18 \\
\hline 2014 & 22 \\
\hline 2015 & 7 \\
\hline
\end{tabular}




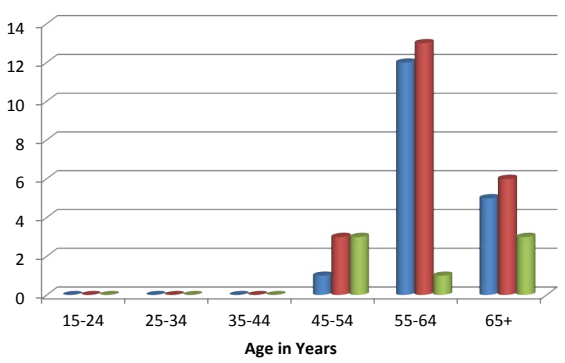

- 2013

- 2014 $\square 2015$

Figure 1. The number of patients with lung cancer by age group. In the period time of 20132015 , the risk for lung cancer increases with age and the most frequently affected age group is 5564 years old.

over 65 years old. In 2015, 3 patients are 45 54 years old, 1 patient is 55-64 years old and 3 patients are over 65 years old.

From 47 patients 45 are males $(96 \%)$ and 2 are females (4\%), as can be seen in FIGURE 2. In 2013, 18 patients are males. In 2014, 20 patients are males and 2 are females. In 2015, 7 patients are males. So men are most affected by lung cancer.

From these 26 patients or $55 \%$ are from the city and 21 patients or $45 \%$ are from village, as shown in FIGURE 3. In 2013, 12 patients are from the city and 6 patients are from village. In 2014,8 patients are from the city and 14 patients are from village. In 2015, 6 patients are from the city and 1 patient is from village. So the lung cancer is more spread in urban areas than rural ones.

As can be seen in FIGURE 4, 30 patients are smokers (64\%) and 17 patients are non-smokers (36\%). In 2013, 10 patients are smokers and 8 patients are non-smokers. In 2014, 15 patients are smokers and 7 patients are non-smokers. In 2015, 5 patients are smokers and 2 patients are non-smokers. For smokers the risk is much higher, while for non-smokers the risk is lower.

As can be seen in FIGURE 5, 17 patients are non-smokers, 4 patients have smoked $<30$ pack years, 7 patients have smoked 30 to 60 pack years and 19 patients have smoked $>60$ pack years. In 2013, 1 patient have smoked $<30$ pack years, 2 patients have smoked 30 to 60 pack years and 7 patients have smoked $>60$ pack years. In 2014, 2 patients have smoked $<30$ pack years, 4 patients have smoked 30 to 60 pack years and 9 patients have smoked $>60$ pack years. In 2015, 1 patient have smoked $<30$ pack years, 1 patient have smoked 30 to 60 pack years and 3 patients have smoked $>60$ pack years.

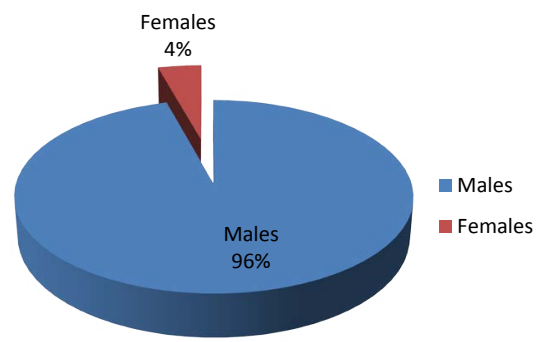

Figure 2. Lung cancer (males vs. females). In the period time of 2013-2015, lung cancer was found more often in men than in women.

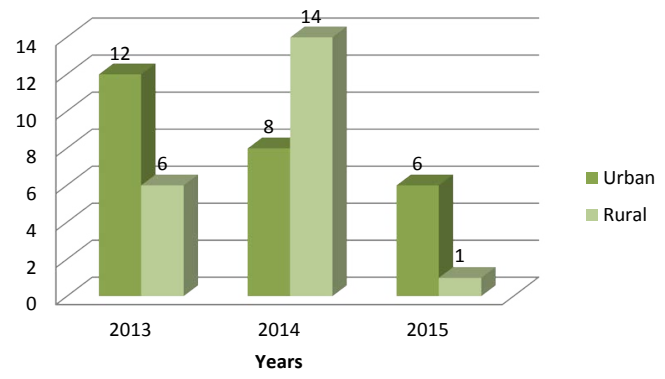

Figure 3. The number of patients with lung cancer by urban/rural residence. In the period time of 2013-2015, the lung cancer is more spread in urban areas because the air pollution level is high.

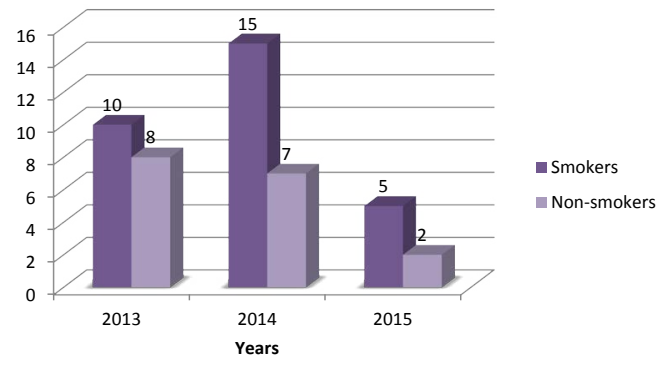

Figure 4. The number of patients with lung cancer by smoking status. In the period time of 2013-2015, lung cancer mainly affects smokers versus non-smokers.

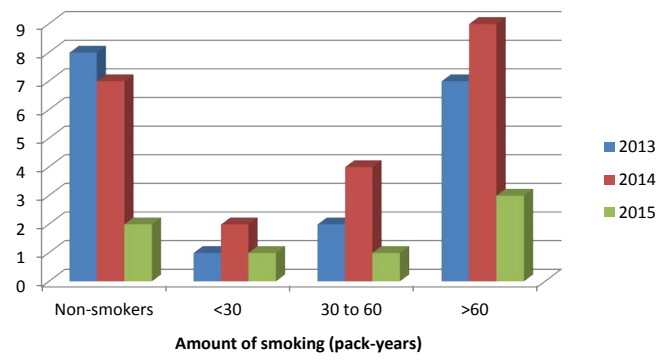

Figure 5. The number of patients with lung cancer by the amount of smoking (pack-years). The risk of lung cancer increases with the number of cigarettes smoked.

From 47 patients 33 have a family history of cancer and 14 have no family history of 


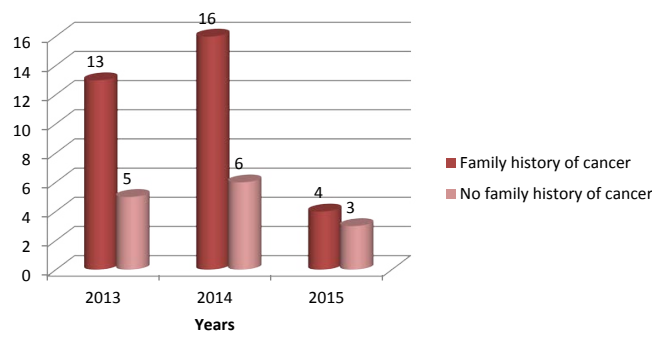

Figure 6. The number of patients with or without a family history of cancer. In the period time of 2013-2015, the incidence of lung cancer is greater in those with a family history of cancer.

cancer, as can be seen in FIGURE 6. In 2013, 13 patients have a family history of cancer and 5 patients have no family history of cancer. In 2014, 16 patients have a family history of cancer and 6 patients have no family history of cancer. In 2015, 4 patients have a family history of cancer and 3 patients have no family history of cancer [16].

\section{Discussion}

Our results demonstrate an increase in risk of lung cancer in individuals aged 55-64 years. We also found a significantly increased risk associated with family history regardless of age-at-onset.

Lung cancer was found more often in men than in women. The major risk factor for lung cancer emerging from this study is active cigarette smoking. Most patients over 64\% smoked cigarettes; however, the disease also affected non-smoking patients [16].

Lung cancer is a multifactor disease, even though the smoking habits in the population exceed all other factors in importance. The most widespread and strongest risk factors in addition to active smoking include radon in indoor air (residential houses and workplaces) and exposure to carcinogenic substances at work. Other factors, such as passive smoking and general air pollution, may also contribute because they affect large segments of the population over a long period of time, but the individual increase in risk is relatively modest.

\section{Conclusion}

Based on the date obtained from patients treated for lung cancer we conclude that the most frequently affected age group is 55-64 years old. The most affected sex is male. The lung cancer is more spread in urban areas than rural ones because the air pollution level in urban areas is high. We conclude that lung cancer mainly affect smokers versus non-smokers. The risk for lung cancer increases with age and cumulative exposure to tobacco smoke. Our results suggest that those with a family history of cancer are more likely to acquire lung cancer themselves.

\section{REFERENCES}

1. Ozols RF, Herbst RS, Colson Yl et al. Clinical cancer advances 2006: Major research advances in cancer treatment, prevention and screening a report from the American Society of Clinical Oncology. J. Clin. Oncol. 25, 46 (2007).

2. Ferlay J, Autier P, Boniol M et al. Estimates of the kanch incidence and mortality in Europe in 2006. Ann. Oncol. 18, 581 (2007).

3. Sano H, Marugame T. International comparisons of cumulative risk of lung cancer, from cancer incidence in five continents. Jpn. J. Clin. Oncol. 35, 334 (2006).

4. Wakelee HA. Chang ET, Gomez SL et al. Lung cancer incidence in never smokers. J. Clin. Oncol. 25, 472 (2007)

5. Vineis P, Alavanja M, Buffer $\mathrm{P}$ et al. Tobacco and cancer: Recent epidemiological evidence. J. Natl. Cancer. Inst. 96, 99 (2004).

6. Alberg AJ, Samet JM. Epidemiology of lung cancer. Chest. 123, 21-49 (2003).

7. Flehinger BJ, Melamed MR, Zaman MB et al. Early lung cancer detection: results of the intial (prevalence) radiologic and cytologic screening in the Memorial Sloan-Kettering study. Am. Rev. Respir. Dis. 130, 555 (1984).

8. Matakidou A, Estein T, Houlston RS. Systematic review of the relationship between family and lung cancer risk. Br. J. Cancer. 93, 825 (2005).

9. Hwang SJ, Cheng LS, Lozano G et al. Lung cancer risk in germ line p53 mutation carriers: association between an inherited cancer predisposition, cigarette smoking and cancer risk. Hum. Genet. 113, 238 (2003).

10. Landi S. Gemignani F, Canzian F et al. DNA repair and cell cycle control genes and the risk of young-onset lung cancer. Cancer. Res. 66, 11062 (2006).

11. Whitrow MJ, Smith BJ, Polotto LS et al. Environmental exposure to carcinogens causing lung cancer: Epidemiological evidence from the medical literature. Respirol. 8, 513 (2003).

12. Bosken $\mathrm{CH}$, Wei Q, Amos CI et al. An analysis of DNA repair as a determinant of survival in patients with non-small cell lung cancer. J. Natl. Cancer. Inst; 94, 1091 (2002).

13. Valaitis J, Warren S, Gambel D. Increasing incidence of adenocarcinoma of the lung. Cancer. 47, 1042 (1981).

14. Noguchi M. Morikawa A, Kawasaki M et al. Small adenocarcinoma of the lung. Histologic characteristics and prognosis. Cancer. 75, 2844 (1995).

15. Kish JK, Ro JY, Ayala AG et al. Primary mucinous adenocarcinomas of the lung with signet- ring cells. A histochemichal comparison with signet-ring cell carcinomas of other sites. Hum. Pathol. 21, 459 (1989).

16. Katzenstein AL, Prioleau PG, Askin FB. Ihe histologic spectrum and significance of clear cell change in lung carcionoma. Cancer. 45, 943 (1980). 